\title{
L'Interconnector : un réseau de transmission de gaz européen. Approche lexicologique comparative du français et de l'anglais des réseaux et contrats gaziers
}

Jacqueline Percebois

\section{(2) OpenEdition}

\section{Journals}

Édition électronique

URL : http://journals.openedition.org/asp/2446

DOI : $10.4000 /$ asp. 2446

ISBN : $978-2-8218-0380-0$

ISSN : 2108-6354

\section{Éditeur}

Groupe d'étude et de recherche en anglais de spécialité

\section{Édition imprimée}

Date de publication : 1 décembre 1999

Pagination : 223-252

ISSN : 1246-8185

\section{Référence électronique}

Jacqueline Percebois, «L'Interconnector : un réseau de transmission de gaz européen. Approche lexicologique comparative du français et de l'anglais des réseaux et contrats gaziers », ASp [En ligne], 23-26 | 1999, mis en ligne le 01 septembre 2011, consulté le 19 avril 2019. URL : http:// journals.openedition.org/asp/2446; DOI : 10.4000/asp.2446

Ce document a été généré automatiquement le 19 avril 2019

Tous droits réservés 


\section{L'Interconnector : un réseau de transmission de gaz européen. Approche lexicologique comparative du français et de l'anglais des réseaux et contrats gaziers}

Jacqueline Percebois

1 En économie de l'énergie, les réseaux ont une place prépondérante : réseaux d'électricité, réseaux de gaz, réseaux d'éclairage public, de chauffage urbain... L'électricité et le gaz sont typiques des network industries, les industries de réseau (autres exemples: l'eau, les télécommunications).

2 Nous avons choisi d'aborder les réseaux par le biais de l'Interconnector, réseau européen de gaz reliant Bacton (UK) sur la côte du Norfolk et Zeebrugge (Belgique), mis en service le $1^{\text {er }}$ octobre 1998.

\footnotetext{
Completion of the line is due by October 1998, after which Britain's gas reserves and pipeline grid will be connected with the grids of continental Europe for the first time. (1) ${ }^{1}$

It will be the first direct physical link of its kind between the UK and continental Europe. (2)
}

Ce réseau a une réalité physique puisqu'il s'inscrit dans l'espace géographique européen ; c'est aussi un réseau immatériel avec sa gestion électronique par le screen-based gas trading market de Bacton qui régit ainsi les relations offre-demande inter ou transnationales.

4 Nous présenterons d'abord le corpus sélectionné : revues spécialisées en anglais et en français, textes institutionnels, ouvrages sur le gaz, mais aussi nombreux sites Internet. 
La présentation du corpus nous permettra de préciser le cadre dans lequel s'inscrit l' Interconnector et d'en citer les principaux champs lexicaux. Nous nous poserons la question: ces premiers éléments d'analyse nous permettent-ils d'affirmer que ce corpus est bien représentatif d'une langue de spécialité?

L'intérêt se portera alors sur sa terminologie spécifique. À partir de l'étude des éléments du corpus concernant le réseau physique et immatériel, existant et évolutif, de l' Interconnector, nous nous proposons de faire une analyse comparée d'éléments du lexique de l'anglais et du français de l'Interconnector trans-Manche.

6 Nous aborderons certaines spécificités terminologiques présentant un intérêt particulier pour le chercheur en langue de spécialité, des KWICs selon l'acronyme employé par John Sinclair : «This acronym stands for Key Word In Context » (1991: 173), dans un mode d'étude toutefois différent puisqu'il s'agissait alors d'un "popular type of computer-generated concordance ». Nous étudierons les acceptions de ces unités lexicales, les circonstances qui ont déterminé leur emploi et leur évolution. Nous mettrons en évidence les nombreux cas d'emprunt de termes anglais en français ainsi que les procédés d'économie linguistique qui caractérisent la terminologie de ce réseau de gaz (troncation, siglaison, unités de mesure).

7 Le terme «Interconnector » est le plus souvent conservé, avec l'orthographe anglaise et la majuscule, en français. Toutefois, nous avons trouvé dans le corpus quelques cas de traduction ainsi que l'abandon de la majuscule, par exemple « l'interconnecteur gazier». Par ailleurs le français, comme l'anglais, utilise aussi d'autres dénominations qui se veulent explicatives : «le gazoduc Interconnector, le nouveau réseau de transit gazier d'ouest en est», ou restrictives: "le gazoduc trans-Manche, the cross-Channel Interconnector », comme nous le constaterons avec le relevé des collocations (Annexe 4).

À l'évidence, ces précisions peuvent éviter des confusions avec d'autres gazoducs ou d'autres «Interconnector»: les deux Irish Interconnectors: the UK-Republic of Ireland Interconnector et the UK-Northern Ireland Interconnector.

Enfin, « Interconnector » est une société : Interconnector (UK) Ltd. (Annexe $3: 1$ ).

The Interconnector is a transportation facility for gas shippers who buy capacity, and is capable of transporting 20 billion cubic metres of gas per annum from the UK to Continental Europe. There is also the ability to reverse the flow and transport gas to the UK at a capacity of $8.5 \mathrm{bcm}$ p/a. (3)

10 Par l'emploi des noms Interconnector, transportation facility, gas shippers; des verbes: transporting, transport, ainsi que des unités de mesure abrégées ou non : $b c \mathrm{~cm} / \mathrm{a}$. ou billion cubic meters per annum, cette citation est tout à fait représentative du lexique du corpus.

\section{Le corpus}

\section{1. Principes de sélection}

11 Une fois défini le sujet de cette présentation, se pose la question du corpus. Sur ce point, nous nous référerons à la démarche de Jacky Martin dans son article « Du bon usage des corpus dans la recherche sur le discours spécifique» (1997). Nous nous intéresserons à une 
[...] forme de linguistique qu'on pourrait qualifier, faute de qualificatif plus attesté, de «linguistique de corpus» pour laquelle seules comptent les régularités du corpus, les collocations et les combinaisons variables entre sémantique et syntaxe. (1997 : 75)

\section{en langue spécifique » :}

Dès l'instant où c'est la spécificité qui est en question, et non la représentativité comme dans les corpus généralistes, l'étoffement du corpus s'arrête dès que cette spécificité cesse de se manifester de façon significative. Ce point est atteint dès l'instant où l'ajout de nouveaux textes cesse d'introduire de nouveaux termes spécifiques [...]. (ibid. : 79)

L'événement référentiel est ici la mise en service du cross-Channel Interconnector, European gas network, entre Bacton et Zeebrugge. Cet événement référentiel concentrera autour de lui des éléments disparates rassemblés en une speech community. Admettant, avec Christopher Gledhill (1997: 87), que « [...] pour bien exploiter le corpus spécialisé, il faut le relier à sa communauté ", nous allons nous y attacher, notamment à l'aide d'Internet. (Annexe 1)

15 En effet, outre le recours à des revues spécialisées en anglais ou en français, des textes institutionnels et des ouvrages relatifs aux réseaux de gaz, Internet s'est révélé une mine d'informations irremplaçable. Toutefois, les sites Internet étant susceptibles d'être constamment modifiés, nous soulignerons le problème posé par la citation de ces références. En passant par Altavista et les mots clés interconnector gas network, apparaît immédiatement la question : «Spell check: did you mean inter connector? » ce qui atteste de la non-lexicalisation actuelle du terme Interconnector, avec ou sans majuscule.

Les sites Internet peuvent être classés en plusieurs catégories: les constructeurs du réseau, les institutions, les compagnies gazières, les cabinets de consultants, les revues spécialisées dans le domaine du gaz, uniquement ou entre autres énergies (pétrole, électricité, charbon...), à travers lesquels va se dessiner cette communauté.

17 C'est l'accès direct à certains sites, peu nombreux, qui permet de trouver des informations postérieures à la mise en œuvre du réseau en octobre 1998 et en premier lieu le site de Interconnector (UK) Ltd <http://www.iuk-isis.com>. Une autre source d'information, les e-mails de certains organismes (Annexe 1), a été exploitée de façon marginale en raison du faible taux de réponses ou de l'intérêt limité des réponses.

Les différents éléments de ce corpus constituent un " canal de transmission de données » selon les termes de Maria Térésa Cabré (1998: 122), et nous nous référerons à son ouvrage La Terminologie pour répondre à la question: peut-on valider ces éléments de corpus comme représentatifs d'une langue de spécialité?

\section{2. Éléments du corpus et langue de spécialité}

19 Pour en juger, citons Maria Teresa Cabré et examinons notre corpus à la lumière des éléments mentionnés : 
[...] est spécialisé (dans le sens large) tout type de discours qui s'éloigne des caractéristiques générales par un des éléments suivants (1998:122)

- « le sujet» : the Interconnector gas network.

- "les caractéristiques spécifiques des interlocuteurs»: les États, les institutions, les compagnies gazières, les économistes spécialistes de l'énergie et, de façon plus sélective, des réseaux de gaz;

- «les caractéristiques spécifiques de la situation de communication » : La communication se produit dans un contexte professionnel, scientifique ou technique; la terminologie, plus formalisée que la langue courante, est normalisée pour éviter les ambiguïtés. En effet, les langues de spécialité servent à la communication entre spécialistes, les producteurs de communication spécialisée ; dans ce cas, les auteurs s'adressent à des pairs, aux membres d'une communauté scientifique ou technique. Les langues de spécialité servent aussi à l'information ou la formation d'autres individus, les récepteurs de communication spécialisée, techniciens ou étudiants de troisième cycle par exemple, ce qui peut impliquer des niveaux différents de technicité.

- «la fonction de communication » est de type informatif, fonction référentielle; il s'agit de transmettre de l'information : « conveying information objectively » (Thorne 1997 : 434).

- «le canal de transmission des données » : ici les diverses «voies» de transmission définies dans le corpus ; il s'agit de communication écrite sur papier ou Internet.

\section{Le lexique} corpus, nous mettrons en évidence des unités lexicales : lexical units ou, amalgame par troncation du premier terme, lexunits (Summers 1997: 7) et leurs collocations ou cooccurrences.

\subsection{Unités lexicales simples ou complexes}

À l'origine du lexique se trouve "l'unité fonctionnelle mémorisée en compétence » de Bernard Pottier (1985 : 265), ou « l'unité lexicale mémorisée » de Jean Tournier (1993 : 11). C'est la lexie qui sera simple, composée ou complexe. En appliquant la classification de J. Tournier au corpus de l'Interconnector gas network, nous remarquerons :

- un petit nombre de lexies simples primaires, constituées d'«un seul élément lexical autonome » ex. : link, gas, grid;

- de très nombreuses lexies simples dérivées avec affixes ex. : Interconnector, interconnection, offpeak, offshore, oversupply, overhang, underground, subsurface, infrastructure;

- de très nombreuses lexies composées, "formées d'au moins 2 éléments lexicaux autonomes » (Tournier 1993 : 12) ; ex. : gas-fired, grid-fed, screen-based ; gas bubble, spot market, capacity trading, end users.

La phraséologie composée des lexies prépositionnelles et des lexies complexes :

- nombreuses lexies prépositionnelles, "[...] c'est-à-dire un groupe introduit par une préposition » (Tournier 1993 : 12) ; ex. : under contract, on a long-term basis ; 
- nombreuses lexies complexes : les phrases lexicalisées : take or pay, gas-on-gas competition, pipeline-on-pipeline competition, dash-for-gas. lexical patterns attachés à ces termes, les dénominations et leur co-texte, c'est-à-dire les collocations (Annexe 4).

\subsection{Structuration du lexique}

\subsubsection{From all-purpose language to topic-specific terms}

En fonction de la spécificité du document considéré, nous avons constaté des variantes dans la terminologie dite topic-specific. Cette observation est fondée sur le principe de structuration pyramidale de la langue de spécialité dû à Michel Perrin. Selon ce principe, la langue courante, abondante, constitue la base de la pyramide tandis que la terminologie spécifique au domaine étudié, dite topic-specific, restreinte, constitue le sommet (Percebois 1996 : 113-114).

Le corpus retenu pour étudier la terminologie économique du réseau de gaz Interconnector, fait apparaître cinq strates (de bas en haut) :

- all-purpose language: en particulier, termes relatifs au réseau, à l'interconnection: connection, transportation, link ; transport, liaison. to connect, interconnect, transport, link ; relier, acheminer, transporter.

- specific use of general language : vocabulaire général à orientation scientifique et technique : trade, facility, commissioning; contracter.

- general scientific language: vocabulaire économique général: supply and demand, monopoly, comparative advantage, barriers to entry, overheads; la terminologie empruntée à la théorie des jeux : players, commitment, bid, risk.

- subject-specific language: vocabulaire de l'économie de l'énergie: utilities, marché spot, transactions spot, swaps. spot est lexicalisé en français en économie de l'énergie, swap est en cours de lexicalisation en économie générale. (lexicalisation : "The process whereby a coinage becomes an established lexical item »). 
- topic-specific terms : terminologie du réseau de gaz Interconnector : price cap, capacity, shippers , TOP, TPA, etc.

$$
\text { maintenant. }
$$

\subsubsection{Différents champs lexicaux}

Dans cette strate supérieure, nous distinguons différents champs lexicaux (lexical ranges) :

- Le projet: L'Interconnector est en premier lieu et au départ un projet : la terminologie du projet sera donc prépondérante : planning, evaluation, feasibility study, Interconnector study group, design, implementation ; en phase de réalisation.

As a result of these political, commercial and operational pressures, Tim Eggar, then energy minister for the UK government, initiated further development of the gas interconnector project. (4)

- L'installation du réseau, c'est-à-dire sa construction, avec pour l'essentiel le lexique de l'ingénierie, de la technologie (ex. : site Jan de Nul) : dredging vessels, landfall dredging, offshore technology, engineering, hydraulic testing; terminal, compression.

- La gestion du réseau : TOP, TPA, trading : demand and supply, risk management and hedging tools, consumers or users.

- Les contrats: ex. sites de consultants tels que WoodMackensie; gas export contracts, contracted amounts, the contract year, TOP contracts.

- La libéralisation du marché du gaz. Les titres sont évocateurs : «Implied in the UK; will it reach Europe through the Interconnector?»; "From the monopoly buyer, transporter and marketer through to the fully competitive gas market for all users ", «A more competitive pricing regime in the marketplace ». (Annexe $3: 7$ )

The connection of gas grids of the UK and mainland Europe by the BactonZeebrugge Interconnector is more than just a physical connection. It also links a progressive and competitive market in the UK with a developing and yet to liberalise market in mainland Europe. (4)

- Unités lexicales faisant référence à la durée : les échéances dans la phase de construction de l'Interconnector network, les stipulations des contrats : shorter term sales; on a long term basis, in the immediate future, until 2000, throughout 1993 and 1994, since its early development, over the years. (Annexe $3: 12,14,16-18$ )

L'Interconnector est ainsi un exemple des « [...] interface areas where the different fields fuse » (Varantola $1986: 11)$.

\subsection{Key words in context avec équivalents français}

Nous étudierons des «ordinary words used with a specific meaning». Ces mots ordinaires prennent des sens spécifiques dans le domaine étudié :

- la spécificité du cas Britannique : gas bubble, dash-for-gas, beach price ;

- la gestion du réseau : regulator, shippers, trading, capacity, third party access, contracts, end-users ;

- les incidences de la Directive Gaz Européenne: single buyer, eligible consumers, eligible customers. 
Dans ces divers cas, les termes, d'importance capitale dans le contexte, ont des équivalents français. Nous les classerons en deux grandes parties.

\subsubsection{Le fonctionnement du réseau}

contrôle d'un regulator, par des shippers selon divers principes d'accès au réseau, parmi
lesquels TPA.

Gas bubble : la bulle gazière (Annexe $3: 3,25$ )

34

[...] «bulle» de gaz (ou surplus de la capacité de production par rapport aux débouchés existants). (Terzian 1997 : 92) En fait, il faut distinguer la «bulle» de British Gas et la «bulle » gazière britannique dans son ensemble.

Dash-for-gas : le mot d'ordre de la politique énergétique britannique depuis 1990 :

The major development on that side of the industry since 1990 has been a "dash-for-gas" (5)

The fuel displaced in this "dash-for-gas" has been mainly coal, although oil usage has also declined (6)

Cela a entraîné « the unrelenting march of gas » :

From 1990 to 1995, the share of natural gas in electricity generation increased from under $1 \%$ to $16 \%$ and is expected to grow to $38 \%$ by the year 2000 (7)

Beach price, prix «à la plage », dénomination due à l'installation des terminaux gaziers sur les côtes où arrive le gaz en provenance des plates-formes offshore.

Gas is brought to the 6 reception points (called beach terminals) by gas producers operating rigs in about 100 fields beneath the sea around the British Isles. After treatment, [...] it is transported into the Transco pipeline system.(13)

«beach to meter» est d'ailleurs le titre d'une page du site Internet de Transco (cf. 2.5.2) présentant son réseau de distribution. En revanche, le «border price of gas in continental Europe ", c'est par exemple le prix du gaz russe arrivant à la frontière française. (beach/ border price : voir aussi Annexe $3: 3,25$ ).

Regulator : le régulateur, l'autorité de régulation, les instances de régulation (l'adjectif français régulateur se rendant par regulatory). Notons que l'équivalent français de l'anglais regulation est ici régulation: on parlera de cadre de régulation, fonction de régulation

La régulation porte sur le contrôle des prix, le comportement des acteurs sur le marché, la sécurité et l'environnement :

The principal function of the regulator, and the regulatory function which will be required for many years to come, is that of price control.

[...] the role of the regulator is to ensure that the monopolist doesn't engage in anti-competitive behaviour. (8)

Le regulator n'est pas une personne mais une entité « the Director General of Gas Supply and his staff (Ofgas) ». Cette entité est personnifiée : il n'est question que de l'indépendance, l'autonomie, le pouvoir réel, l'avis, le statut, les décisions du régulateur. Le régulateur de 
l'industrie du gaz britannique, c'est Ofgas, dénomination qui sera étudiée dans les amalgames (2.5.2).

Par ailleurs, le régulateur local devra compter avec une autorité supérieure au niveau européen, «some high level regulation from the commission » :

The EU gas directive also sees the need for local regulation by member states and for some high level regulation from the commission. The challenge will be to manage the framework between the local regulator and the Commission. (4)

41 Shippers (gas suppliers) = chargeurs, transporteurs; $c$ f. 9 " gas shippers who buy capacity" (Annexe $3: 4,27$ ).

Transco... transports and stores gas for its customers, the 40 or so companies (known as shippers) through a gas transportation network...across the UK. (13)

Le pouvoir des transporteurs s'est amenuisé, du fait des surcapacités de transport, de l'interconnexion croissante des réseaux, et de l'imposition de la séparation (« unbundling ») juridique entre transport et commercialisation (décret 636, 1992). (Muluala, 1997 : 203)

En mentionnant transporteurs, surcapacités de transport, interconnexion des réseaux, unbundling, qui sera étudié dans les emprunts (2.4), la citation ci-dessus est particulièrement représentative du lexique du corpus.

End-users : utilisateurs finals, usagers finals. The large end-user market, ce sont les «gasfired power stations, industrial customers such as ICI » (9).

In December 1997, European energy ministers agreed a gas directive which will open up the European gas market to competition in gradual stages from 2000. Large end-users will be able to shop around for the cheapest gas prices, improving their competitiveness. And from 1 October 1998, the UKContinent Interconnector gas pipeline will pump UK gas to mainland Europe, enabling European end-users to buy UK gas directly from North Sea producers, and to benefit from the UK's cheaper prices. (9) (Voir aussi Annexe $3: 4,15)$

44 Capacity trading : Capacity c'est la capacité de transport de gaz disponible (Annexe $3: 6$, $13,18)$. Trading c'est le négoce de ce gaz qui se fait à travers un réseau électronique, screen-based trading ou electronic trading (Annexe 3:5, 6, 26).

The transportation company can also facilitate the development of a capacity trading market by providing an electronic bulletin board (EBB) facility for its customers and for the industry as a whole. (2)

$\mathrm{Au}$ départ l'Interconnector ne doit gérer qu'une export capacity, capacité ou potentiel d'exportation, c'est-à-dire une forward flow capacity, dans le sens UK-Europe, mais une reverse flow capacity est prévue :

The export capacity from Bacton to Zeebrugge (forward flow capacity) is up to twenty billion cubic meters $(\mathrm{bcm})$ per annum, this equates to approximately eight billion therms per annum, which is roughly equivalent to $20 \%$ of annual UK demand. The import capacity from Zeebrugge to Bacton (reverse flow capacity) will be eight and a half bcm (approximately 3.4 billion therms). (2) 
$\mathrm{n}$ 'avons pas trouvé en français de traductions des dénominations forward flow capacity et reverse flow capacity mais des adaptations : " capacité nord-sud » et "capacité sud-nord ». L'Interconnector est d'abord « exportateur de gaz », indique-t-on en français, tout en mentionnant «la réversibilité ultérieure du gazoduc » ou "la deuxième vie de l'Interconnector, lorsque la canalisation servira à importer du gaz au Royaume-Uni » (Terzian 98 : 107-108).

Autre nuance dans l'emploi de capacity c'est celle qui est réservée par les chargeurs sous forme d'entry capacity ou d'exit capacity :

In order to deliver gas into the gas transportation system it is necessary for each shipper to book entry capacity to get gas into the NBP. Entry capacity has to be booked in 12-month tranches [...]. Similarly, if a shipper wishes to offtake gas from the NBP exit capacity has to be booked. Exit capacity is also booked in 12-month tranches (4)

Enfin, avec gas trading hub, nous introduisons le terme hub qui sera vu dans les emprunts (2.4) :

A gas trading hub is either a physical location on a gas supply system, where a number of pipelines converge, probably in the vicinity of a local market, together with gas storage or it is a theoretical point on a system where gas is traded, such as the NBP. (4) (voir aussi Annexe $3: 6,22-24$ )

Third party access : accès des tiers au réseau TPA (UK) = ATR (Fr). Il s'agit d'unité lexicale complexe : ici le sens de l'unité lexicale est bien le résultat de la combinaison du sens des trois morphèmes constitutifs, le français étant toutefois plus explicite : là où l'anglais dit le plus couramment third party access et parfois to the network, le français dira toujours accès des tiers au réseau et parfois ajoutera de transport. (cf. Annexe $3: 7-10$ ). Nous avons même souvent trouvé la collocation "accès des tiers au réseau de transport moyennant péage » :

Les réformes des marchés gaziers anglo-saxons ont entraîné l'introduction de la concurrence au niveau de la commercialisation du gaz par le biais de l'accès des tiers au réseau de transport (ATR) moyennant péage. (Muluala 1997 : 215)

Outre les différences dans l'expression, se rencontrent parfois des différences dans la conceptualisation, les deux phénomènes pouvant même être combinés. Nous noterons ainsi les problèmes signalés à propos de third party access par Jonathan Stern, l'auteur d'un livre consacré à cette question : Third Party Access in European Gas Industries :

There are substantial problems and confusion -even among Englishlanguage speakers - as to its meaning and the differences between TPA and other terms. This is partly because different terminology is used in North America and partly because the terms are inadequately defined by those using them. (1992: 21)

Ici il existe une variation dans l'espace, ou diatopique, de la langue de spécialité. Nous avons également noté l'emploi de neg TPA model, neg étant la troncation de negotiated; le français quant à lui n'utilise pas ici la forme tronquée mais ATR négocié.

51 En revanche, en ce qui concerne regulated Third Party Access, ou regulated TPA, la troncation n'a été rencontrée ni en anglais ni en français : ATR réglementé ou ATR régulé. (Annexe $3: 8-10$ ) 
52 Nous retrouverons l'ATR dans le cadre des emprunts (2.4) car sa gestion occasionne en français l'emploi de loan-words: common carrier, common carriage, open access, qui n'ont jamais été remis en question.

\subsubsection{Les contrats}

53

Accords et contrats abondent dans notre corpus (cf. Annexe 3 : 11-22). L'observation du lexique les concernant permet d'en relever différents types assortis de nombreuses collocations relatives à leur durée, aux volumes de gaz impliqués et à la couverture des risques. Le site Internet de l'Interconnector (UK) Ltd nous révèle qu'une alliance contract strategy a existé dès l'origine du projet de l'Interconnector afin de le mener à bien :

The $£ 450$ million scheme posed a major engineering challenge to all those involved, but the adoption of an alliance contract strategy has helped ensure the project's success. (3)

La maintenance du réseau est aussi gérée par Transco et Distrigaz sous contrat (Distrigaz est belge ; à ne pas confondre avec Distrigas, UK) :

Terminal operations and maintenance are managed under contract by Transco in Bacton and by Distrigas in Zeebrugge. (3)

55 À partir du lancement des premiers contrats, nous retrouvons dans le corpus le lexique de base des contracts (Annexe 4 : II) ; par exemple : to negotiate gas contracts, deals signed so far, to enter into a contract, the contracted amounts; négocier/signer un contrat, le lancement de ce contrat, etc.

Nous devons ici souligner des emplois particuliers du verbe contracter en français, dont voici deux exemples :

Producteurs et acheteurs ont alors les moyens de contracter directement entre eux. (Muluala 1997 : 197)

Dans ce cas, il s'agit d'un emploi intransitif du verbe contracter; or, en français contracter est toujours transitif.

... 2 milliards de $\mathrm{m} 3 \mathrm{du}$ gaz d'Elgin-Franklin ont été contractés par Gaz de France pour être livrés en France à partir de l'an 2000, via l'Interconnector. (10)

Ici il est donné au participe passé contractés le sens de stipulés par contrat qu'il n'a pas en français ; par assimilation flexionnelle cet emploi en français ressemble beaucoup aux contracted amounts, collocation fréquente dans les contrats.

... the «take or pay contracts» that are the norm in continental Europe ... require buyers to pay for gas even if market conditions mean it no longer needs the contracted amounts. (4)

ou contracted for export :

... Up to August 1998, approximately ten bcm of gas has already been contracted for export from the UK to begin 1st October 1998. (2). 
dans cette terminologie usuelle, nous trouvons de nombreuses locutions exprimant des conditions temporelles : « over a seven-year period ... » ou les volumes « contractés »: « Export contracts totalling $48 \mathrm{bn}$ cubic metres of gas have been signed so far for the interconnector ... ».

61 CRTA, capacity reservation and transportation agreement, est le contrat réservant une capacité de transport par les shippers. Outre ces emplois, le corpus met en évidence des spécificités, les future contracts, contrats futures en français :

le premier contrat futures annoncé par l'IPE ne doit porter que sur des transactions/livraisons à la plage ou à l'entrée du réseau et qualifié de FOB pour cette raison. (Terzian1998: 105).

Premier type : le contrat à la plage, à l'entrée du réseau ou FOB

l'IPE a lancé un contrat futures du type CIF, ainsi qualifié parce qu'il porte sur des volumes de gaz livrés après transport... Ce contrat CIF s'appelle National Balancing Point Contract (NBP Contract), en référence à un point géographique notionnel déjà utilisé par le marché spot. L'objectif est d'offrir aux opérateurs un outil de négoce électronique permettant de gérer le risque prix sur une période de 12 mois. (Terzian 1998 : 105)

Deuxième type : contrat de type CIF ou contrat NBP, en anglais : NBP contract $=$ National Balancing Point contract. Ce sont des cas particuliers de FOB contracts et CIF contracts. (Pour NBP, voir aussi Annexe $3: 6,26-27$ )

Nous constatons déjà ici de nombreux emprunts, effectifs bien que non indispensables.

Enfin, BP Gas Marketing a lancé les OTC contracts « un modèle standard de contrat NBP qui s'opère de gré à gré (ou OTC), par opposition au contrat NBP de l'IPE » (Terzian, 1998 : 105).

65 La référence aux contrats futures, les contrats à terme, introduit le lexique de la couverture de risques; en effet ce sont des « contracts that hedge against spot market risks " (Hoster, p. $70 \mathrm{n}^{\circ}$ 26) ex. : "gérer leurs risques prix» "tout en se couvrant physiquement en livraisons », « tous les instruments modernes de négoce et de gestion du risque ", ou encore :

Interconnector va établir une liaison physique entre le Continent Européen et un marché déréglementé -celui de la GB-... où l'on dispose d'une panoplie d'instruments de négoce électronique ou OTC, offrant notamment des possibilités de gérer le risque prix. (Terzian $1998: 107)$

Enfin se détache la terminologie des take or pay contracts : des stranded contracts: contrats échoués ou « overhang of unexpired take or pay contracts» (Annexe $3: 21$ ). Les contrats take or pay seront abordés parmi les emprunts.

Dans les contrats commerciaux certaines dénominations sont liées à la Directive Gaz, the EU gas directive. Parmi elles, l'acheteur unique : the single buyer

... étant la personne morale qui est « responsable de la gestion unifiée du système de transport et/ou de l'achat et de la vente centralisés de l'électricité ». (Batail $97: 124)$

... ou du gaz. 
Par ailleurs, nous avons constaté l'importance de l'adjectif eligible, qualifiant alternativement customers ou consumers.

... les États membres publient, avant le 30 janvier de chaque année, les critères de définition des clients éligibles. (Batail $97: 125$ )

La formule retenue par la directive gaz: une définition des consommateurs éligibles: à savoir tous les producteurs d'électricité à partir de gaz (cogénérateurs inclus) ainsi que les clients dépassant un seuil de consommation par site. Ce seuil est fixé à 25 millions de m3 (environ 20000 tep) à l'entrée en vigueur de la directive (en pratique en 2000), puis 15 millions cinq ans plus tard (en pratique 2003), puis 5 millions dix ans plus tard (en pratique 2008). (11)

Chaque producteur devra trouver par lui-même des demandeurs d'électricité éligibles avec lesquels négocier un contrat commercial; il lui faudra aussi s'arranger avec les réseaux d'interconnexion ou d'alimentation directement concernés pour la fourniture des "services d'accès» correspondants. (Glachant 1997 : 166)

\subsection{Les emprunts}

Lexical borrowing, c'est le processus d'emprunt; "emprunt» désigne également en français le mot emprunté à une autre langue, en anglais : loan-word. Les emprunts sont souvent dus aux transferts scientifiques et technologiques entre pays de langues différentes.

Tant qu'il n'est pas assimilé, ou au moins en voie d'intégration, c'est-à-dire tant qu'il est perçu par l'usager comme un élément étranger, un emprunt constitue ce que l'on appelle un xénisme. (Tournier $1993: 148$ )

Nous constaterons que dans la plupart des cas ces emprunts demeurent des xénismes pour les usagers extérieurs à la communauté de discours définie par le corpus. La première forme qui est utilisée pour dénommer un concept est généralement perçue comme la plus naturelle et, par conséquent, on ne peut facilement la modifier. Seule une intervention concertée peut arriver à changer les attitudes.

71 Pour OTC (Annexe 2: 40, 42) et Network Code, l'emprunt, constaté le plus souvent, est facultatif : en effet il est possible d'avoir recours à des équivalences "de gré à gré ", "Code de réseau ».

Pour OTC, nous lirons : « contrat de gré à gré ou OTC », « une panoplie d'instruments de négoce électronique ou OTC ».

73 Pour le Network Code, il est tentant de citer Lehmann (1998:6) pour qui « Xénisme est réservé à l'emprunt qui correspond à une réalité étrangère (apartheid, toundra) ». Cette dénomination sera conservée en français :

Le 26 février 1996, British Gas TransCo. et les chargeurs ont signé un Network Code qui fixe les règles du jeu dans l'organisation des approvisionnements journaliers et qui vise à permettre à des vendeurs concurrents et à leurs chargeurs de jouir d'un accès égal au système -monopolistique- de gazoducs de British Gas. (Terzian $1998: 103)$

ou, rarement, traduite :

... Mais le lancement de ce contrat a été différé par l'IPE en attendant le règlement des problèmes pratiques nés de l'application du Code de réseau par BG TransCo. (Terzian $1998:$ 104)

Par contre l'emprunt est permanent pour hub, price cap, unbundling et take-or-pay. 
D'après l'Oxford Advanced Learner's Dictionary, un hub est «the central point of activity, interest or importance »; dans notre corpus, le hub, c'est le lieu « where several pipeline operators interconnect »; c'est aussi le hub notionnel dit national balancing point ou NBP (NBP : Annexe $3: 24,26-27)$. Ce terme prend ici un intérêt particulier :

Dans un contexte de concurrence exacerbée, le projet Interconnector s'annonçant comme le premier « hub » gazier européen... (Bezzina \& Poudou $98: 410$ ) (Voir aussi Annexe $2: 6,22-24)$

Le price cap est une limite imposée par le régulateur :

La réglementation par le price cap ou rate cap permet au régulateur de contrôler les prix pratiqués par le gazoduc sans avoir à contrôler ses coûts. Le régulateur impose une limite à l'accroissement des prix pratiqués par le monopole. (David \& Mirabel $1998: 428)$

Les charges d'accès acquittées par les expéditeurs sur le réseau Transco sont réglementées par un price cap depuis le 1er octobre 1994. (David \& Mirabel 1998: 428)

Ce nom composé, susceptible d'être qualifié par un adjectif, « price-cap hybride », pourra aussi être utilisé comme adjectif épithète, « réglementation price cap », comme le montre l'exemple suivant:

... Dans la pratique, une solution intermédiaire a été trouvée à travers le « price-cap hybride »... mécanisme qui est une combinaison d'une réglementation price cap avec l'intégration d'un critère de taux de rendement. (David \& Mirabel $1998: 423$ )

Nous avons également trouvé "procédure tarifaire price cap "; price capping se rendra par plafonnement des prix et "plafond de prix » pourrait être proposé comme équivalent de price cap.

La notion de séparation des comptes, c'est l'unbundling comptable. Divers cas d'emplois sont constatés. Nous citerons un premier cas, en 1992, où le terme n'est pas encore parfaitement lexicalisé ; l'auteur (Tournebise 1992: 11) l'emploie dans un titre, entre guillemets, et mentionne sa signification entre parenthèses en sous-titre :

«L'UNBUNDLING »

(désintégration verticale de la production, du transport et de la distribution)

Dans l'exemple suivant, en 1997, l'absence de guillemets indique une lexicalisation achevée, dans le contexte bien évidemment :

... l'unbundling comptable permettra d'identifier les coûts et recettes liés aux différentes fonctions, et les moyens de production d'EDF seront mis en concurrence avec les moyens des producteurs indépendants. (Batail $1997: 129$ )

Nous noterons que,

... dans certains cas, les Français préfèrent employer un emprunt, même lorsque le français dispose d'une dénomination propre. Cela signifie que le critère d'utilisation de mots provenant d'autres systèmes linguistiques n'est pas toujours déterminé par la nécessité. (Cabré 1998 : 228 note 38)

common carrier, common carriage, open access sont des emprunts liés à la gestion de l'ATR :

Le principe de l'ATR se traduit par l'accès des producteurs à l'utilisation des infrastructures selon le principe du «common carrier», moyennant le paiement du coût moyen du transport... (Muluala, $1997: 218$ )

Dans le système du "common carriage ", tous les tiers ont une garantie d'accès au réseau, avec un partage des capacités au prorata en cas de saturation. (Muluala, $1997: 215$ ) 
Ces trois dénominations ne sont jamais traduites en français (Percebois 1993 : 72).

Dans le système de l'«open access ", l'accès est subordonné aux disponibilités en capacités excédentaires et s'effectue sur la base du «premier arrivé, premier servi». Les propositions de la Commission européenne visent un ATR de type «open access» réservé aux gros consommateurs de gaz. (Muluala 1997 : 215) plus haut, et gros consommateurs de gaz, équivalent des large end-users également évoqués plus haut.

\section{take-or-pay}

Le sens d'une unité composée de plus d'un morphème est en principe le résultat de la combinaison du sens de ces morphèmes, bien que cette tendance connaisse des exceptions. (Cabré 1998 : 154)

Take-or-pay est un engagement d'enlèvement minimum de gaz assorti de pénalités. Nous savons que la coordination au moyen de « or » a des implications sémantiques multiples. Ici les pénalités entrent en jeu s'il n'y a pas enlèvement du gaz.

Pour la locution take-or-pay, de nombreuses qualifications nous paraissent adaptées :

- exemple des multiword units incluant des verbes et parfois des connecteurs (autre ex: polluter-pays-principle);

- coordination avec conjonction (Tournier) ; surcomposé à 3 éléments ;

- «syntagme prépositionnel avec valeur adjectivale »(Cabré);

- « syntagme adjectivé » (Martin \& Berthet).

Nous exposerons la grande diversité des usages de cette dénomination, en particulier en tant qu'adjectif : take-or-pay gas/contracts/liabilities... (cf. Collocations Annexe 4).

À cet égard, puisque nous traitons des emprunts, nous devons citer l'adaptation en français de take-or-pay depletion contracts par contrat take-or-pay du type depletion, exemple incontestable de xénisme au sens emprunté plus haut à Jean Tournier. le recours à une périphrase ; bien sûr il s'agit ici de convention puisque les termes anglais take-or-pay ne donnent pas totalement la clé de la signification de cette locution et du sigle correspondant. Il serait donc possible par convention d'avoir recours à une locution et un sigle français opérant de même, take-or-pay n'étant accessible qu'à ceux qui détiennent la connaissance du concept et du contexte.

L'évolution dans le temps du lexique est dite variation diachronique. Une première intégration du terme anglais dans le contexte français pourra faire place à un équivalent français, souvent à l'instigation du ministère concerné. Il existe donc des différences dans le temps au niveau de l'expression. Nous citerons l'adaptation approximative de take-orpay en 1992 dans un texte du Conseil économique et social : "paye même si on n'enlève pas ", ceci étant une bizarrerie avouée par les guillemets, l'unité lexicale anglaise d'origine n'ayant pas été contestée en contexte français à ce jour. 


\subsection{L'économie linguistique}

88 Le corpus révèle un recours fréquent à l'économie linguistique par l'emploi de sigles ou d'amalgames incluant des troncations. Selon Tournier le coût de l'utilisation d'un mot « a deux aspects, le coût articulatoire et le coût mémoriel » (1993: 138).

Or, une des lois constantes qui régissent l'activité humaine en général est la loi du moindre effort. Appliquée au langage, elle se manifeste par le fait que l'homme a naturellement tendance à réduire le coût, c'est-à-dire à limiter son effort à ce qui est nécessaire et suffisant à la transmission d'un message. (93:139)

\subsubsection{La siglaison}

L'usage de sigles, abbreviations ou letter-words en anglais, entraîne une réduction articulatoire considérable.

Le sigle se prononce lettre par lettre lorsqu'il ne respecte pas la contrainte morpho-

phonique imposée aux mots proprement dits (Tournier 1993 : 142)

La liste des principaux sigles se trouve en Annexe 2. Nous ferons ici quelques remarques concernant la siglaison et certains sigles ou acronymes. Le recours à ce procédé peut entrainer parfois des accumulations excessives, évidemment hermétiques pour le noninitié, par exemple :

Fin 1997, BP Gas Marketing lançait... un modèle standard de contrat NBP qui s'opère de gré à gré (ou OTC), par opposition au contrat NBP de l'IPE. (Terzian, $1998:$ :105)

le Conseil a décidé..., de prévoir des dérogations à l'ATR en cas de risque de TOP pour les opérateurs gestionnaires de réseau. (4)

Le sigle devient acronyme, c'est à dire se prononce comme un mot, «lorsqu'il constitue un ensemble correspondant à un modèle morpho-phonique existant ou possible " (Tournier 1993 : 142) ; par exemple, dans notre corpus :

ISIS : Interconnector Shippers Information System, système d'information des chargeurs de l'Interconnector.

I(UK) also operates the 24-hour Interconnector Shippers Information System (ISIS) which receives shippers' daily nominations, aggregates them and instructs the terminal operators accordingly. It subsequently allocates quantities once they have been shipped. (3)

93 ISIS est un exemple d'acronyme lexico-ludique. En effet, Isis étant une déesse égyptienne, épouse d'Osiris à qui elle rendit la vie, mère d'Horus, type de l'épouse et de la mère idéales, la dénomination ISIS semble être un gage de fiabilité de ce système d'information.

Autre exemple d'acronyme lexico-ludique dans le secteur gazier britannique: CATS: Central Area Transmission System, le système de pipelines qui transporte le gaz du bloc-J en mer du nord britannique (Terzian 1998 : 99).

Nous avons rencontré plusieurs cas d'homonymie parmi les sigles du corpus :

- PPP : Pool Purchase Price; dans d'autres contextes économiques, PPP pourra avoir deux autres sens: Polluter Pays Principle, le principe du pollueur payeur et Purchasing Power Parity, la parité des pouvoirs d'achat.

- LDCs : Load Duration Curves... à ne pas confondre avec less developed countries.

- OTC, Over-The-Counter, qui signifie aussi : Organization for Trade Cooperation. 
Mais ce phénomène est relativement rare: en gênant la communication, il

compromettrait l'intérêt même de la réduction du signifiant. (Tournier 1993 : 143)

Réseau), nous remarquerons des différences dans la qualification des sigles en anglais et en français. L'économie linguistique représentée par le sigle est renforcée en anglais par la troncation postérieure (back clipping) de "negotiated", mais non de "regulated»; en revanche, le français utilise les deux (ou trois) adjectifs sans troncation:

neg TPA ATR négocié

regulated TPA ATR réglementé, ATR régulé

[...] dans l'ATR réglementé, l'accès au réseau fait l'objet de tarifs affichés; dans l'ATR négocié, la rémunération de l'accès au réseau est établie au cas par cas. (Batail $97:$ 124) (Voir aussi Annexe $3: 8$-10)

Enfin, comme nous avons pu le constater dans les extraits cités et pourrons le faire avec les références complémentaires de l'Annexe 3 et la liste de l'Annexe 2, les unités de mesure abondent dans ce corpus, que ce soit dans la gestion du réseau ou les contrats.

\subsubsection{L'amalgame}

L'amalgame est un processus de composition qui se distingue de la composition par juxtaposition par le fait que ses éléments constituants ne sont pas juxtaposés mais " télescopés ", c'est-à-dire emboîtés l'un dans l'autre. (Tournier 1993 : 82)

L'amalgame inclut souvent la troncation (clipping) qui «peut entrainer une réduction importante du coût articulatoire, les formes tronquées dépassant très rarement deux syllabes » (Tournier, 1993 : 139). C'est le cas de celles que nous avons rencontrées dans ce corpus.

OFGAS, pour Office of Gas Supply, exerce la fonction de régulateur de l'industrie du gaz britannique :

the independent government watchdog for the gas industry (4).

Il doit déterminer la formule tarifaire de BG et veiller au bon fonctionnement de l'ATR. (David \& Mirabel 1998 : 427) transcription « of gas » : propre au gaz, du domaine gazier. Ofgas apparait aussi sous les traits d'un vrai « watchdog », Gus, sur le site Internet (12).

OFFER, ou Offer, est un surcomposé, un acronyme composé de la $1^{\text {re }}$ syllabe du $1^{\text {er }}$ élément 'office' et des initiales des deux derniers éléments 'electricity' et 'regulation'; soit: troncation postérieure ou apocope (angl. back clipping) et sigle.

The whole electricity sector including the market mechanism is overseen by the Office of Electricity Regulation (OFFER) whose Director General is a political appointee. (Hoare 1997 : 75) 
Ofgas and Offer are in the process of merging to form a new regulatory office for the gas and electricity industry. The new organisation will be run by the Management Committee Structure ... which was announced on Thursday 11 March 1999.

... mais pour l'instant nous ne savons pas sous quelle dénomination apparaîtra ce nouveau regulatory office.

TRANSCO, ou TransCo, est un acronyme composé de la première syllabe de 2 mots: troncations postérieures de transport company.

[...] the BG business unit in charge of operating and maintaining the pipeline and storage facilities.

[...] Transco is meant to be a guardian of the industry's daily balance. (4)

Transco delivers gas from the beach to the meter utilising its network of 170,000 miles of pipeline to 19.5 million homes and businesses around the country. (13)

"from the beach to the meter» est une collocation qui sert de titre à l'une des pages Internet du site Transco (13).

\section{Conclusion}

François Gaudin (1993: 77) a défini «une lexicologie aux fonctions sociales» en constatant que

[...] c'est dans le cadre d'institutions administratives, politiques ou économiques, que la terminologie s'est développée. [...] En fait, le développement de la terminologie ne répond pas simplement à une demande sociale, il n'intervient que lorsqu'existent des enjeux historiques, politiques ou économiques.

L'enjeu est ici la transmission du gaz européen. La mise en parallèle des lexiques anglais et français à partir du corpus sélectionné pour étudier ce phénomène, par l'exemple de l' Interconnector gas network, a révélé à quel point les remarques de Gaudin s'appliquent à ce cas, en particulier dans l'adaptation de la terminologie française à partir de l'anglais. Il s'agit d'un réseau européen, dépendant d'administrations publiques et privées de différents pays, mettant en jeu des langues différentes, ici l'anglais et le français, mais dépendant aussi d'une Directive européenne représentant une autorité supérieure. Par ailleurs, nous avons déjà constaté la forte influence de l'anglais dans la terminologie de l'énergie en général (Percebois 1993).

Au cours de cette étude, nous avons rencontré des illustrations des trois ressorts de la création lexicale exposés par Tournier (1993: 168) : la connaissance et la communication, la loi du moindre effort et la pulsion ludique.

En effet, le corpus nous a permis de mettre en évidence des unités lexicales indispensables à la communication entre spécialistes et à la formation des récepteurs de communication spécialisée, ces communications et formations passant parfois par des emprunts, plus fréquents dans ces documents spécialisés que dans le lexique général. Est également plus fréquent le recours à la siglaison, dans ses divers aspects, représentatif de 
cette loi du moindre effort. Quant à la pulsion ludique, nous l'avons reconnue dans certains acronymes, que Michel Petit aime à qualifier de lexico-ludiques.

[...] the interconnectors may have an impact far in excess of their apparent significance. (4)

111 C'est ce que nous constatons dans cette approche lexicologique comparative du français et de l'anglais des réseaux et contrats gaziers en focalisant notre observation sur l'Interconnector trans-Manche. Cette étude nous a permis d'établir une relation entre la fonction de communication de la langue de spécialité, qui sert à transmettre ou échanger des informations sur un thème spécialisé, et les voies de communication offertes par un réseau de transmission de gaz desservant une unité géographique.

\section{BIBLIOGRAPHIE}

Batail, J. 1997. «Le marché intérieur de l'électricité : la négociation de la directive et l'organisation du futur système français ». Économies et sociétés 5-6, 117-136.

Bezzina, Jérôme et J.-Christophe Poudou. 1998. « Régulation et organisation industrielle gazière ». Revue de l'énergie 499, juillet-août-septembre, 408-419.

Bouscaren, C. et F. Lab. 1998. Les Mots entre eux - Words and their Collocations. Paris : Ophrys.

Cabré, Maria Teresa. 1998. La Terminologie, Théorie, méthode et applications. Ottawa : Presses de l'Université d'Ottawa -Armand Colin ; traduit du catalan et adapté par Monique C. Cormier et John Humbley.

Chevalier, Jean-Marie. 1997. « La stratégie des acteurs. La montée des arbitrages interénergétiques ». Économies et sociétés 5-6, 295-311.

David, Laurent et François Mirabel. 1998. « La tarification de l'accès des tiers au réseau - Le cas du gaz ». Revue de l'énergie 499, juillet-août-septembre, 420-438.

Gaudin, François. 1993. Pour une Socioterminologie, des problèmes sémantiques aux pratiques institutionnelles. Rouen : Publications de l'Université de Rouen $n^{\circ} 182$.

Glachant, J.-M. 1997. « Le marché anglais de l'électricité, modèle des échanges européens ?». Économies et sociétés 5-6, 153-170.

Gledhill, Chris. 1997. « Les collocations et la construction du savoir scientifique ». ASp 15-18, 85-104.

Hoare, J. 1997. « Le marché de l'électricité au Royaume-Uni ». Économies et sociétés 5-6, 73-88.

Hoster, Frank, Riechmann, Christoph \& Wallter Schultz. 1997. « The modalities of grid access, Towards an integrated European grid?». Économies et sociétés 5-6, 47-72.

Hudson, R.A. 1996. Sociolinguistics, 2nd ed.. Cambridge : Cambridge University Press.

Laguerre, B. \& D. Haag. 1997. « Quelques remarques sur la régulation du secteur électrique ». Économies et sociétés 5-6, 171-187. 
Lehmann, Alise \& Françoise Martin-Berthet. 1998. Introduction à la Lexicologie - Sémantique et morphologie. Paris : Dunod.

Madden, Mike. The Interconnectors, Financial Times Energy, ISBN 185334-915-1.

Martin, Jacky. 1997. « Du bon usage des corpus dans la recherche sur le discours spécifique ». ASp 15-18, 75-84.

Muluala, Lédy. 1997. « La dynamique de restructuration des activités gazières ». In Énergie et théorie Economique. Paris : Éditions Cujas, 203-254.

Nattinger, James R. \& Jeanette S. DeCarrico. 1992. Lexical Phrases and Language Teaching. Oxford : Oxford University Press.

Percebois, Jacqueline. 1993. «Passage de l'anglais au français en économie de l'énergie : assimilation ou transposition? ». ASp 2, 69-80.

Percebois, Jacqueline. 1996. « Anglais de spécialité en économie de l'énergie : analyse du discours et pédagogie générique ». ASp 11-14, 103-120.

Pottier, Bernard. 1985. Linguistique générale. Paris : Klincksieck.

Radetzki, Marian. 1998. «European Natural Gas : Market Forces Will Bring About Competition In Any Case ». IAEE Newsletter, Third Quarter, 22-27.

Sinclair, John. 1991. Corpus, Concordance, Collocation. Oxford : Oxford University Press.

SPRU. 1998. Energy Programme Newsletter 41, Summer - Science and Technology Policy Research.

Stern, Jonathan. 1993. Third Party Access in European Gas Industries : regulation-driven or market-led? The Brookings Institution.

Summers, Della. 1997. « Corpus lexicography ». Longman Language Review 3, 6-9.

Terzian, Pierre. 1998. Le Gaz naturel, Perspectives pour 2010-2020. Commissariat Général du Plan. Paris : Economica.

Thorne, Sara. 1997. Mastering Advanced English Language. London : Macmillan Master Series.

Tournebise, Alain. 1992. Les services publics du gaz et de l'électricité et le grand marché européen 4204, Conseil économique et social.

Tournier, Jean. 1991. Structures lexicales de l'anglais. Paris : Nathan.

Tournier, Jean. 1993. Précis de lexicologie anglaise, $3^{\mathrm{e}}$ éd. Paris : Nathan.

Varantola, Christa. 1986. «Special language and general language : Linguistic and didactic aspects ». ALSED-LSP Newsletter 9/2, décembre.

Vlaanderen, Paul. 1998. « Energy transit : The multilateral challenge ». IAEE Newsletter, Third Quarter.

\section{ANNEXES}

\section{Annexe 0 : références des passages cités}

(1) www.gasandoil.com

(2) Alliance Gas, sept. 98

(3) www.iuk-isis.com 
(4) www.online.ftenergy.com

(5) www.uow.edu.au/arts/sts/srussell/electricity1999s.html

(6) eande.lbl.gov/National Council/pubs/restdeba.html/

(7) 5 Jan.99, www.foe.co.uk

(8) www.dundee.ac.uk/petroleumlaw/html/article3-15.htm

(9) Petroleum Argus : http://www.saladin.com/sisnew10/page04.htm

(10) www.elf.fr

(11) www.industrie.gouv.

(12) www.ofgas.gov.uk

(13) www.transco-bgplc.com

\section{Annexe 1. Le corpus}

\section{Revues spécialisées en anglais ou en français}

The Energy Journal, IAEE 17/ 2, 1996.

FT Financial Times - Energy : The Interconnectors. 85334915 1, 1998.

International Association of Energy Economists Newsletter, Third Quarter 1998.

Alliance Gas, 1/7, September 1998. The Interconnector : Impact on UK gas prices. Published by Alliance Gas Management Inc, Toronto, Canada.

SPRU Energy Programme Newsletter, Summer 1998, 41 (Science and Technology Policy Research, University of Sussex, Brighton, UK).

Energy Policy, 27/1, January 1999.

Économies et sociétés, série Économie de l'énergie, 5-6/1997 : La déréglementation de l'Electricité : Quels enjeux pour l'Europe ? PUG.

Revue de l'énergie, 499, juillet-août-septembre 1998 : Les mutations de l'industrie électrique et gazière en Europe et en Amérique.

Revue de l'énergie, n 501, novembre 1998.

Textes institutionnels :

UK : Network Code. January 1997. Executive summary sur Internet.

Directive gaz. December 1997. Executive summary sur Internet.

La Politique énergétique de la France : passion ou raison. $n^{\circ} 439$ Les Rapports du Sénat 1997-1998.

Les services publics du gaz et de l'électricité et le grand marché européen. 1992. Rapport présenté par M. Alain Tournebise, Conseil économique et social. Paris : Direction des Journaux Officiels.

\section{Ouvrages}

Stern, Jonathan. 1992. Third Party Access in European Gas Industries.

Terzian, Pierre. 1998. Le Gaz naturel, Perspectives pour 2010-2020. Paris : Economica. 


\section{Sites Internet}

http://www.iuk-isis.com (Interconnector UK Ltd)

Compagnies

http://www.bgplc.com/contact.html

http://www.bgplc.com/business/transco.html

http://www.bgplc.com/world/interconnector.html

http://www.bgplc.com/views/tiscmemo.html

http://www.bgplc.com/databook/interconnector.html

http://www.ofgas.gov.uk

http://www.hemscott.com (BG international.downstream)

http://www.transco-bgplc.com

http://www.centrica.co.uk

http://www.ukbusinesspark.co.uk (renseignements sur Ofgas)

http://www.alliancegas.com

http://www.bp.com/gasinfo/gasactivity.html

http://www.bpamoco.com/_nav/e.mail/

http://www.phenergy.co.uk/egm.htm

http://www-wci-coal.com/gennews3.htm

http://www.elf.fr

http://www.elf.fr/doss/gaz.htm

http://www.elf.fr/us/actu/dernmn/elf14.htm (July 28, 1997)

http://www.ifp.fr/TXT/CO/ARCHIVE/IN112G21.html

http://www.rjb.co.uk

http://www.eyi.com/energy.nsf

Sites gouvernementaux

http://www.accc.gov.au/nem/ch10d.htm (voc. électricité incluant « interconnector ») http://www.eia.doe.gov/cabs/northsea.html (US energy information administration) http://www.industrie.gouv.fr/energie/gaz/se_direc.htm http://www.eande.lbl.gov/National Council/pubs/restdeba.html\#dash Références technologiques http://www.pipe-line.com/contents.html http://www.pipe-line.com/gasfocus.html http://www.offshore-technology.com/contractors/...denul/jan1.html (installation of coffer) 
Contrats

http://www.rike.com/internat.htm (international oil and gas contracts and negotiation) http://www.gasandoil.com/goc/contract/welcome.html http://www.gasandoil.com/goc/news/nte70501.htm http://www.thompson.com/tpg/energy/astr/astrtoc.html (natural gas transportation) http://www.langham.co.uk/mt104.htm (Colloque : Natural Gas Agreements)

Éditeurs ou sites éducatifs

http://www.online.ftenergy.com (Financial Times Energy)

http://www.ogjonline.com (Oil \& Gas Journal)

http://www.dri.mcgraw-hill.com/energy/ukgas.htm

http://www.uow.edu.au/arts/sts/srussell/electricity1990s.html

http://www.dundee.ac.uk/petroleumlaw/html/article3-15.htm

Adresses électroniques d'organismes

info@jandenul.com

jim@rike.com

gasforum@oilmanuk.com (ofgas)

gasnoil@t-online.de

news@energy-gb.com

enquiries.box@gas.co.uk

transco.communications@dial.pipex.com

info@bgplc.com

info@gazdefrance.com

enquiries@langham.co.uk

webmaster@thompson.com

\section{NOTES}

1. Les chiffres entre parenthèses indiquent la source du passage, figurant en annexe 0.

\section{RÉSUMÉS}

En économie de l'énergie, l'actualité récente a mis en lumière l'Interconnector Trans-Manche, réseau de transmission de gaz européen. Nous nous attacherons à faire une approche 
lexicologique comparative du français et de l'anglais des réseaux et contrats gaziers. À travers l'analyse du corpus, nous étudierons les unités lexicales considérées comme mots clés en contexte dans les deux langues, les emprunts et divers procédés d'économie linguistique.

In Energy Economics, recent events have attracted attention to the UK-Continent Interconnector, a European gas transmission network. This paper offers a comparative lexicological approach to gas networks and contracts in English and French. Through the analysis of the corpus, it examines lexical units considered as key words in context in both languages, lexical borrowing and various modes of linguistic economy.

\section{INDEX}

Mots-clés : anglais de spécialité, économie de l'énergie, lexicologie comparative, linguistique de corpus, réseau

Keywords : comparative lexicology, corpus linguistics, energy economics, ESP, network

\section{AUTEUR}

\section{JACQUELINE PERCEBOIS}

Jacqueline Percebois est maître de conférences HDR à la Faculté des Sciences économiques de l'Université Montpellier 1 où elle enseigne en $2^{\mathrm{e}}$ et $3^{\mathrm{e}}$ cycles. Elle a publié L'Anglais de la microéconomie et L'Anglais de la macroéconomie (Éditions Economica). Son domaine de recherche est depuis de nombreuses années la langue de spécialité en économie de l'énergie et de l'environnement. Outre ce thème, elle travaille sur l'anglais de l'économie internationale. Jacqueline.percebois@univ-provence.fr 